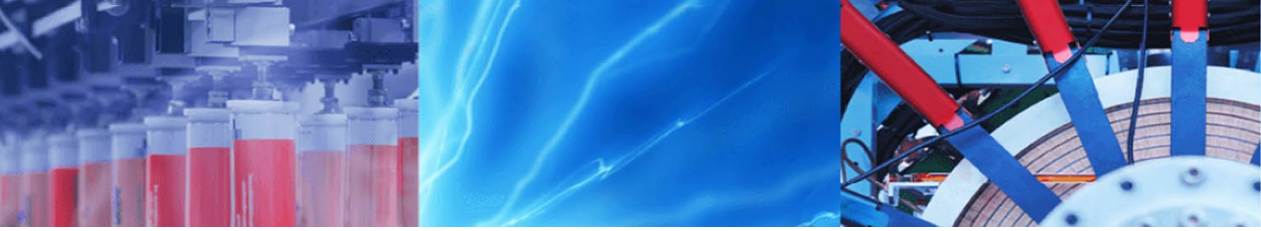

Research Article

\title{
Fault diagnosis of single-point cutting tool using vibration signal by rotation forest algorithm
}

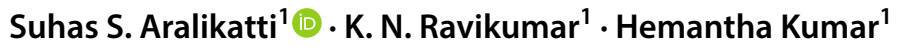

(c) Springer Nature Switzerland AG 2019

\begin{abstract}
In various machining operations, the tool condition monitoring (TCM) is highly necessary to avoid uncertain downtime in production. TCM provides continuously the condition of cutting tool by noticing various parameters such as temperature, acoustic emission and vibration. One of the best ways to monitor the condition of cutting tools for unmanned machining is by observing tool vibration signature. In the present work, vibration signals are acquired from the cutting tool. One healthy state and three faulty conditions of tools are considered for the study. The faulty tools considered in the current study are worn flank, broken tool and extended overhang. The vibration signals of these faulty tool conditions are used to train the machine learning algorithm. Statistical features are extracted from the vibration signal to feed as input to the $J 48$ decision tree. The classifier algorithm used in the current study is rotation forest algorithm. The algorithm uses only significant features which are selected from a decision tree. The algorithm is validated with test dataset to recognize the faulty or healthy state of the tool. It was found that the algorithm could classify the tool condition with $95.00 \%$ classification accuracy.
\end{abstract}

Keywords Tool fault diagnosis · Machine learning technique $\cdot$ Turning operation $\cdot$ Vibration signal $\cdot$ Decision tree

\section{Introduction}

Lathe produces cylindrical parts by shear deformation of the workpiece with relatively high hard single-point cutting tool [1]. The parts produced on lathe find numerous applications in the engineering field - the extent of quality required varies application to application [2]. The desired surface finish on a machined component is achieved by continuous monitoring of anomalies in the cutting process such as tool wear, workpiece hardness and the geometry of the tool [3]. To check the factors influencing the machining process, continuous monitoring of the tool health is necessary. Tool condition monitoring ensures machining takes place as desired without compromise in the quality of the job produced [4]. The online monitoring of the machining process increases the production rate and minimizes the scrap created due to poor machining [5].
Tool condition monitoring (TCM) is a technique to keep a check on tool condition, kind of surface finish produced on the workpiece. With TCM, one can identify anomalies influencing the metal cutting process. TCM was applied to various machining operations such as drilling, milling and turning. Madhusudana et al. $[6,7]$ experimented to diagnose milling tool condition through vibration signals. Histogram features were extracted from vibration signals pertaining to healthy and faulty conditions of the tool. A decision tree was used to capture significant features in the dataset. K-star algorithm was used as a classifier to identify various classes of fault in the milling tool.

Wang et al. [8] studied the application of support vector machine (SVM) in multiclass gear fault diagnosis. Classification accuracy was found to be excellent when training data set and testing data set belonged to identical rotational speed. Since training data set was not available at

Suhas S. Aralikatti, amsuhas@gmail.com | 'Department of Mechanical Engineering, National Institute of Technology Karnataka, Surathkal 575025, Karnataka, India.

SN Applied Sciences (2019) 1:1017| https://doi.org/10.1007/s42452-019-1028-9

Received: 20 December 2018 / Accepted: 31 July 2019 / Published online: 10 August 2019 
all rotational speeds, interpolation and extrapolation of test data were used to help SVM classifier perform fault diagnosis. Ma et al. [9] used the random forest algorithm to predict the tool life of the milling cutter with 315 number of milling test data. The results were compared with other algorithms such as artificial neural network (ANN) and support vector regression (SVR) and random forest (RF). It was found that RF had better tool life prediction accuracy than the rest of two. Bartarya and Choudhury [3] reviewed papers on various machining operations. Kishore et al. [10] designed a magnetorheological damper to control tool vibration during hard turning. The implementation of damper improved the surface finish on the workpiece and reduced tool wear and cutting force. Ventura et al. [11] investigated the development of compressive residual stress on cutting edge and explained the possible cause for the encountered phenomenon. Kuljanic and Sortino [12] introduced torque force indicator (TFD) and normalized cutting force indicator (NCF) as online tool wear indicators of milling process by studying the feature vector parameters of cutting force signal obtained using rotating dynamometer. Dutta et al. [13] reviewed the development of digital image processing technique in tool condition monitoring. Teti et al. [14] presented the keynote paper on past contributions of CIRP in tool condition monitoring, process control, sensor fusion and future challenges, advanced signal processing and recent trends in machining operation. Kothuru et al. [15] performed comparison studies of SVM and convolutional neural network (CNN) technique in the field of machining operation. Progression of wear rate in cutting tool and variation in hardness in the workpiece were analysed by utilizing the above-said techniques as decision-making models.

\section{Methodology}

Various conditions of tool considered for the study are a healthy tool, a tool with breakage, worn flank face and extended overhang. Machining is carried out on a universal lathe machine with these conditions separately. An accelerometer is mounted on the tool holder to acquire the vibration signal. The behaviour of the tool for various tool conditions is recorded by acquiring the vibration signal. The vibrations signals are acquired using a National Instruments data acquisition system (DAQ) and recorded in a laptop for interpretation and post-processing.

The signal is converted into tabular form, and statistical features are extracted by feeding into an analysis of variance (ANOVA) tool. The significant features, which will help in classifying the tool condition, are selected by using a decision tree. The exact classification of tool conditions through the selected significant features is done using rotation forest algorithm. Classification results obtained from rotation forest algorithm is validated using ten fold cross validation method in WEKA software (Fig. 1).

\section{Experimental setup}

Experiments were carried out on all geared precision lathe-panther 1350/2 with cutting parameters set according to tool maker's recommendations with a feed rate of $0.35 \mathrm{~mm} / \mathrm{rev}$ and depth of cut (DOC) of $0.5 \mathrm{~mm}-$ indexable insert used for the hard turning of AISI 1040. The experimental setup comprises lathe, a piezoelectric accelerometer, NI DAQ and a computer with preinstalled LabVIEW software. The workpiece with $60 \mathrm{~mm}$ diameter and $250 \mathrm{~mm}$ length is used. The workpiece is held firmly in the three-jaw chuck and cutting tool secured rigidly into the tool holder. The Piezotronics (make) accelerometer was mounted on the tool post using a magnetic mount. The sampling rate at which the vibration signal acquired was $25 \mathrm{kHz}$. The schematic of the experimental setup is shown in Fig. 2. The actual established experimental setup is shown in Fig. 3.

\subsection{Experimental procedure}

In the present work, AISI 1040 steel is used as work material for the experiment. Coated WC insert used to machine AISI 1040 workpiece. Optimum cutting parameters were

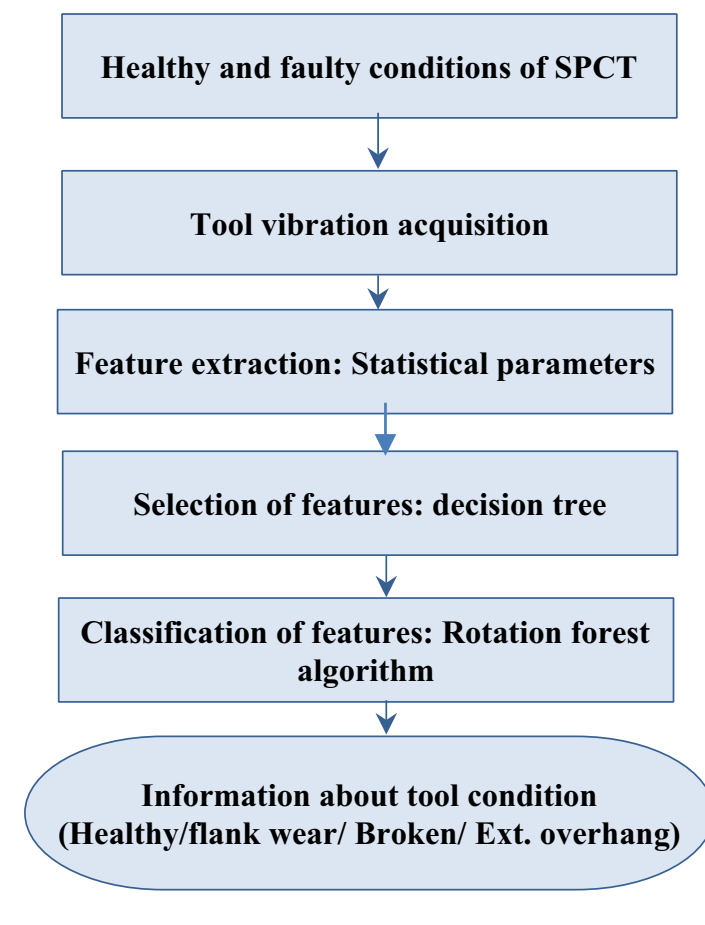

Fig. 1 Methodology followed in tool fault diagnosis 


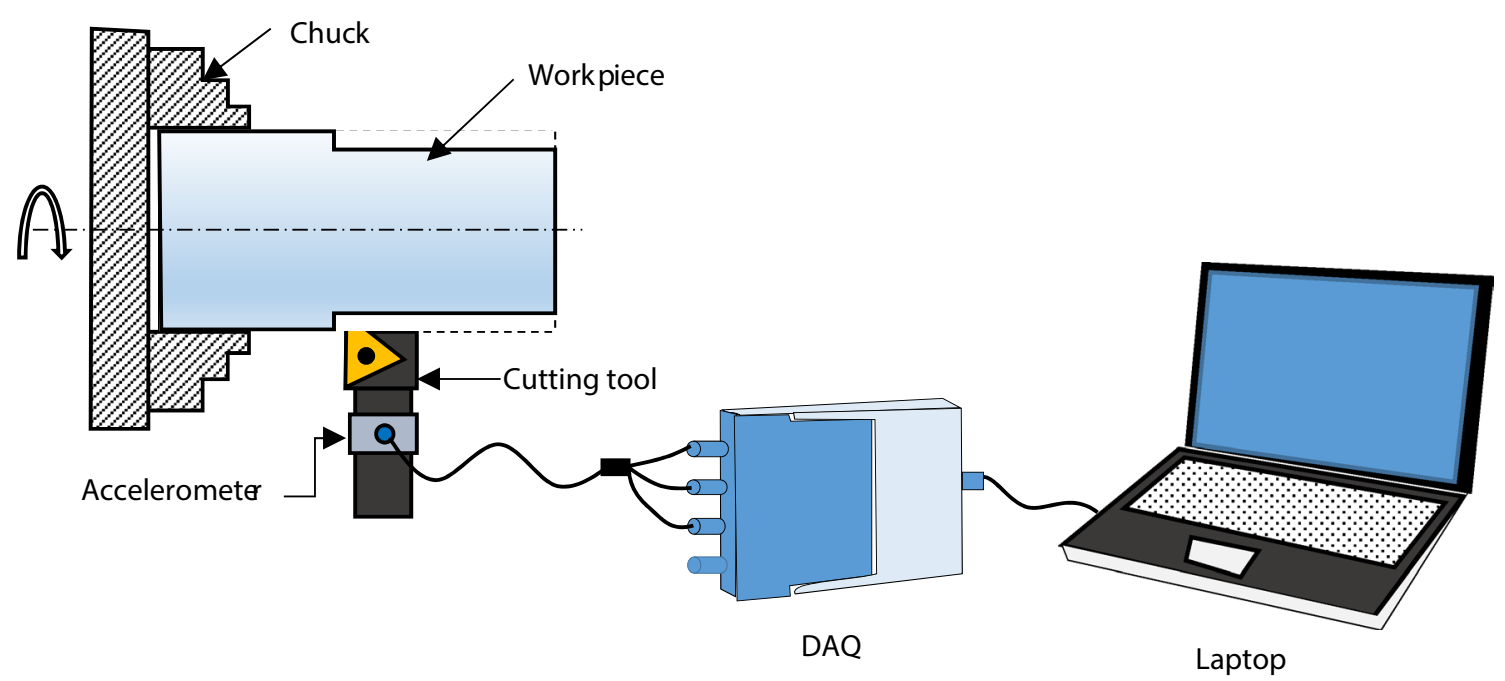

Fig. 2 Schematic representation of experimental setup

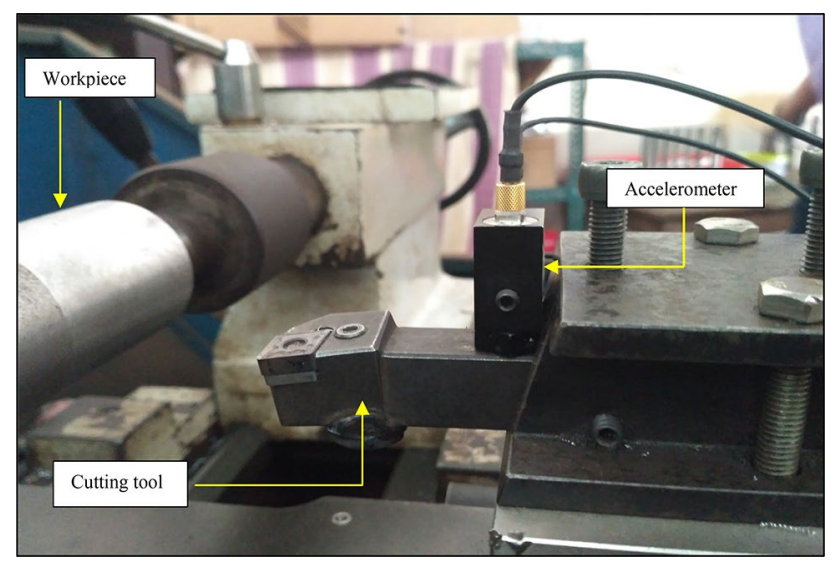

Fig. 3 Image of experimental setup showing accelerometer mounting on tool holder during machining

maintained to ensure no influence of them in fault diagnosis due to tool wear. Specimen preparation is done prior to the experimentation by turning the workpiece to remove the oxide layer and achieve roundness. The machine tool is allowed to stabilize after the start of the machine tool for any random vibration if present $[16,17]$. The different tool conditions considered for the experiment were:

1. A proper mounted but with breakage; labelled as 'breakage'

2. A proper mounted but with flank wear; labelled as 'flank wear'

3. A tool with extended overhang but with the healthy insert; labelled as 'overhang'

4. A proper mounted with healthy insert; labelled as 'proper'

The images of various tool conditions are shown in Fig. 4.

A total number of 120 samples were acquired, with 30 samples for each condition of the tool. The vibration signals corresponding to healthy and faulty conditions are shown in Fig. 5. It is observed that 'maximum' and 'range' for the healthy condition are small and narrow, whereas for the faulty condition, the 'max' and 'range' are more and broad. This graphical representation can differentiate good machining from poor machining but cannot classify the fault. Thus, there is a need to study the vibration behaviour
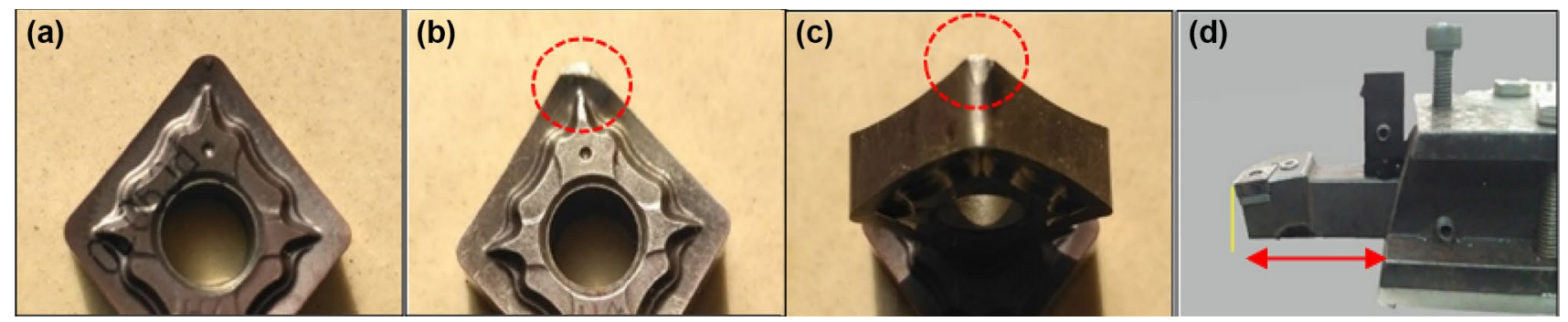

Fig. 4 Pictorial representation of various tool conditions: $\mathbf{a}$ healthy tool, $\mathbf{b}$ tool with breakage, $\mathbf{c}$ worn flank face and $\mathbf{d}$ extended overhang 

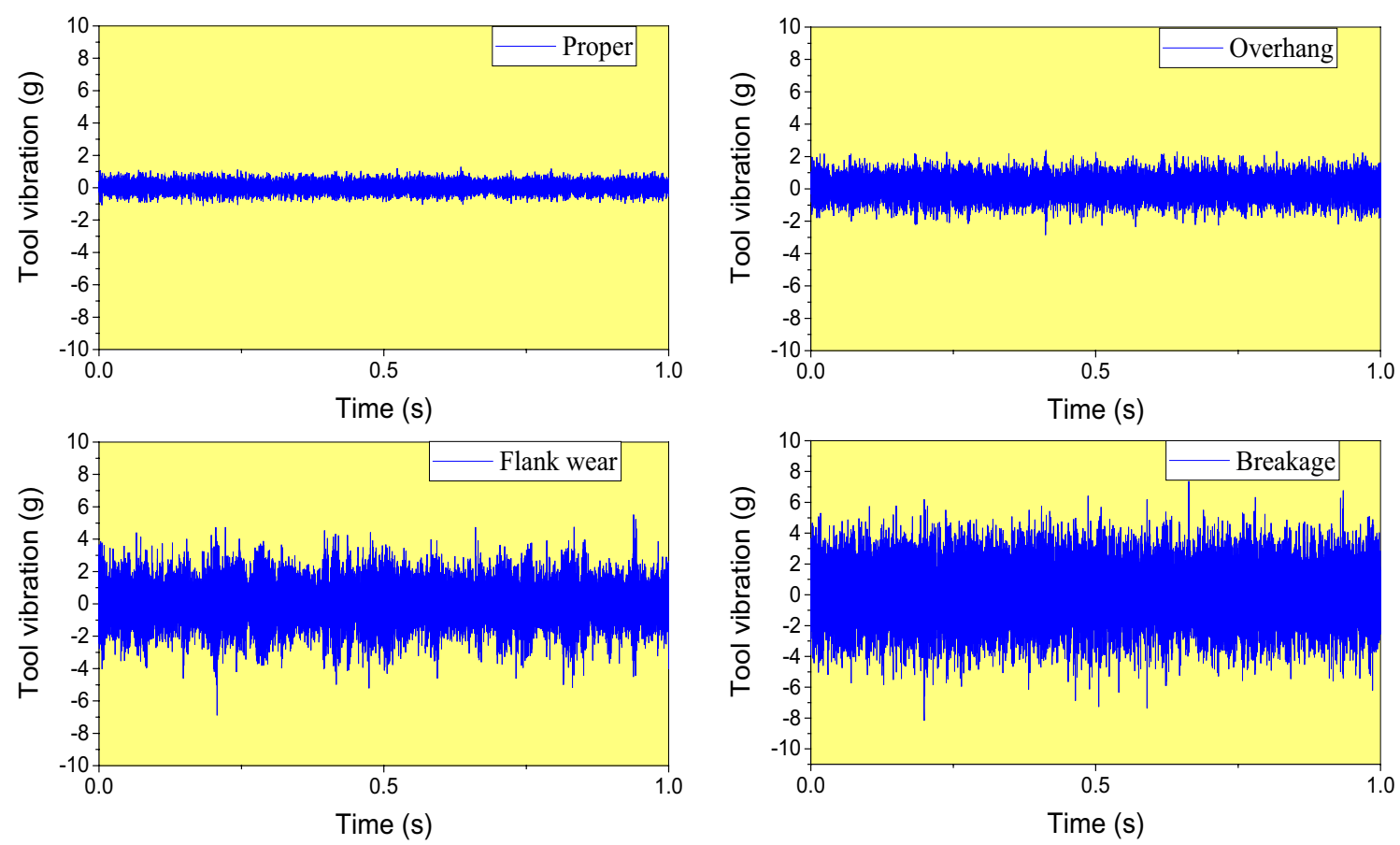

Fig. 5 Time-domain plots vibration signals for various conditions of tool with label on their respective plot

for the various conditions of the tool and classify them accordingly by using a suitable classifier.

\section{Statistical feature extraction}

Statistical feature extraction is one basic signal processing technique which uses mathematical tool to transform raw signals which are nonlinear in nature into informative signatures against the noise. A feature is distinguishing property obtained from a section of a pattern (here vibration signal). Features are extracted to represent the important information hidden in the signal by decomposing the signal. Statistical features extracted in the current study are mean, sum, minimum, maximum, variance, standard deviation, kurtosis and skewness [18]. These features are described in Table 1.

\section{Decision tree as tool for feature selection}

A decision tree is a pictorial representation of an algorithm as a tree-like model with conditional control statement visible. The decision tree has nodes, branches and roots to represent the classification of samples according to heuristic model. A decision will have a single root node for complete training set of data. A new node is added to the tree for every partition [6]. The detailed steps involved in developing the decision tree are listed below [19]:
1. The tree starts with a node representing the training samples of data collected

2. If the samples are all of the same class, then they are labelled as leaf

3. Otherwise, the algorithm will divide the samples into individual classes based on the entropy-based measure known as information gain by discretizing every attribute to select the optimal threshold.

4. To create the branch, samples are portioned for each best discrete interval

5. The algorithm uses the same steps mentioned above repeatedly to form the decision tree

6. This repetition process to form the tree stops once one of the following criteria is met

a. When all the samples of a node given belong to one class

b. When there is no attribute remaining to partition the samples

c. When samples get exhausted for the branch test attribute

\section{Rotation forest algorithm}

Rotation forest (RF) goals at the construction of precise and varied classifiers. RF is a type of ensemble technique, which can train ' $L$ ' number of decision trees distinctly. It transforms the data set while retaining all the information 
Table 1 Statistical features and their definition

\begin{tabular}{|c|c|c|c|}
\hline SI. no. & Terminology & Statistical formula & Description \\
\hline 1 & Mean & $\mu_{1}=\bar{X}=\frac{1}{n} \sum_{i=1}^{n} X_{i}$ & $\begin{array}{l}\text { A dimensional quantity that measures the central tendency of the } \\
\text { distribution (a measure of location) }\end{array}$ \\
\hline 2 & Variance & $\mu_{2}=\sigma^{2}=\frac{1}{n} \sum_{i=1}^{n}\left(X_{i}-\bar{X}\right)^{2}$ & $\begin{array}{l}\text { A dimensional quantity that measures spread about the mean or } \\
\text { power of fluctuation from the mean }\end{array}$ \\
\hline 3 & $\begin{array}{l}\text { Higher statistical } \\
\text { moments about } \\
\text { mean }\end{array}$ & $\mu_{k}=\frac{1}{n} \sum_{i=1}^{n}\left(X_{i}-\bar{X}\right)^{k} \quad$ where $k=3,4,5$ & Higher moments \\
\hline 4 & Standard deviation & $\sigma=\sqrt{\mu_{2}}=\sqrt{\frac{1}{n} \sum_{i=1}^{n}\left(X_{i}-\bar{X}\right)^{2}}$ & $\begin{array}{l}\text { A dimensional quantity that measures the variability of the distribu- } \\
\text { tion or fluctuation from the mean }\end{array}$ \\
\hline 5 & Skewness & $\chi=\frac{\mu_{3}}{\sigma^{3}}=\frac{\frac{1}{n} \sum_{i=1}^{n}\left(X_{i}-\bar{X}\right)^{3}}{\sigma^{3}}$ & $\begin{array}{l}\text { A nondimensional feature that measures the degree of asymmetry } \\
\text { of the distribution (or shape of the distribution) about the mean }\end{array}$ \\
\hline 6 & Kurtosis & $\kappa=\frac{\mu_{4}}{\sigma^{4}}=\frac{\frac{1}{n} \sum_{i=1}^{n}\left(X_{i}-\bar{X}\right)^{4}}{\sigma^{4}}$ & $\begin{array}{l}\text { A nondimensional feature that reflects the peakedness of the } \\
\text { distribution }\end{array}$ \\
\hline 7 & Standard error & $Y=\sqrt{\frac{1}{(n-2)}\left[\sum(y-\bar{y})^{2}-\frac{\left[\sum(x-\bar{x})(y-\bar{y})\right]^{2}}{(x-\bar{x})^{2}}\right.}$ & $\begin{array}{l}\text { The standard error is a measure of the amount of error in the esti- } \\
\text { mation of } y \text { and for an individual } x \text { in the regression }\end{array}$ \\
\hline
\end{tabular}

in the set. Ensemble classifier unites the predictions of several classifiers instead of only one classifier. Because ensemble reduces the variance and bias, it is essential to alter the data set bagging, boosting or learning method to create different classifiers.

Let $X=\left[x_{1}, x_{2} \ldots x_{n}\right]^{\top}$ be a data point with $\mathrm{n}$ features and $X$ be an $N \times n$ matrix containing the training examples. $Y=\left[y_{1}, y_{2} \ldots . . y_{n}\right]^{\top}$ is class label with c classes. Assume that true class labels of all training examples are also provided. The ensemble vector is $D=\left\{D_{1}, D_{2} \ldots D_{L}\right\}$, where $L$ is the number of different classifiers in the ensemble and $F$ is the feature set. All classifiers can be trained in parallel. The detailed study has been carried out by Rodríguez et al. [20]

\section{Results and discussion}

\subsection{Statistical feature extraction for the experimental dataset}

Statistical features were extracted by analysis of variance (ANOVA). The labelled data set containing vibration signal information in tabular form is given to the software. Only tangential vibration was considered for the study which was dominant than the rest of other directions. A one second data of vibration signal of a class (tool condition) had 25,600 data points. There were thirty second data for each class; there were four classes in the experiment. One second data set is reduced to 12 statistical features.

Random tree (RT) algorithm is one variant of decision trees. There were 12 statistical features, which were fed to the RT algorithm. Figure 6 shows the decision tree which used seven features, namely SE of mean, standard deviation, variance, kurtosis, sum, maximum and skewness for the classification of features. These features serve as input to the classification algorithm. Looking at the decision tree diagram, one can observe that 'overhang' class has been classified with two features; 'proper' class classified with three features; 'flank wear' classified with five features; and 'breakage' classified with seven features. The class, which uses more features, has data similarity with other classes.

\subsection{Tool fault classification using rotation forest algorithm}

Twelve statistical features pertaining to vibration signal are obtained for all 120 samples and given as input to the feature selection algorithm (decision tree). The decision tree used standard error, standard deviation, variance, kurtosis, sum, maximum and skewness as significant features to classify the different classes of data [21].

The confusion matrix of the rotation forest algorithm is presented in Table 2. The matrix in the table comprises four rows and four columns. The number of rows depends on the number of instances considered. The confusion matrix obtained from the decision tree is discussed below [22]:

- The diagonal elements give information about correctly classified instances in the confusion matrix.

- The first number in the first line corresponds to 'proper' state being classified correctly as 'proper condition'.

- The second number in the first line signifies the misclassified instance belonging to 'proper' as flank wear 


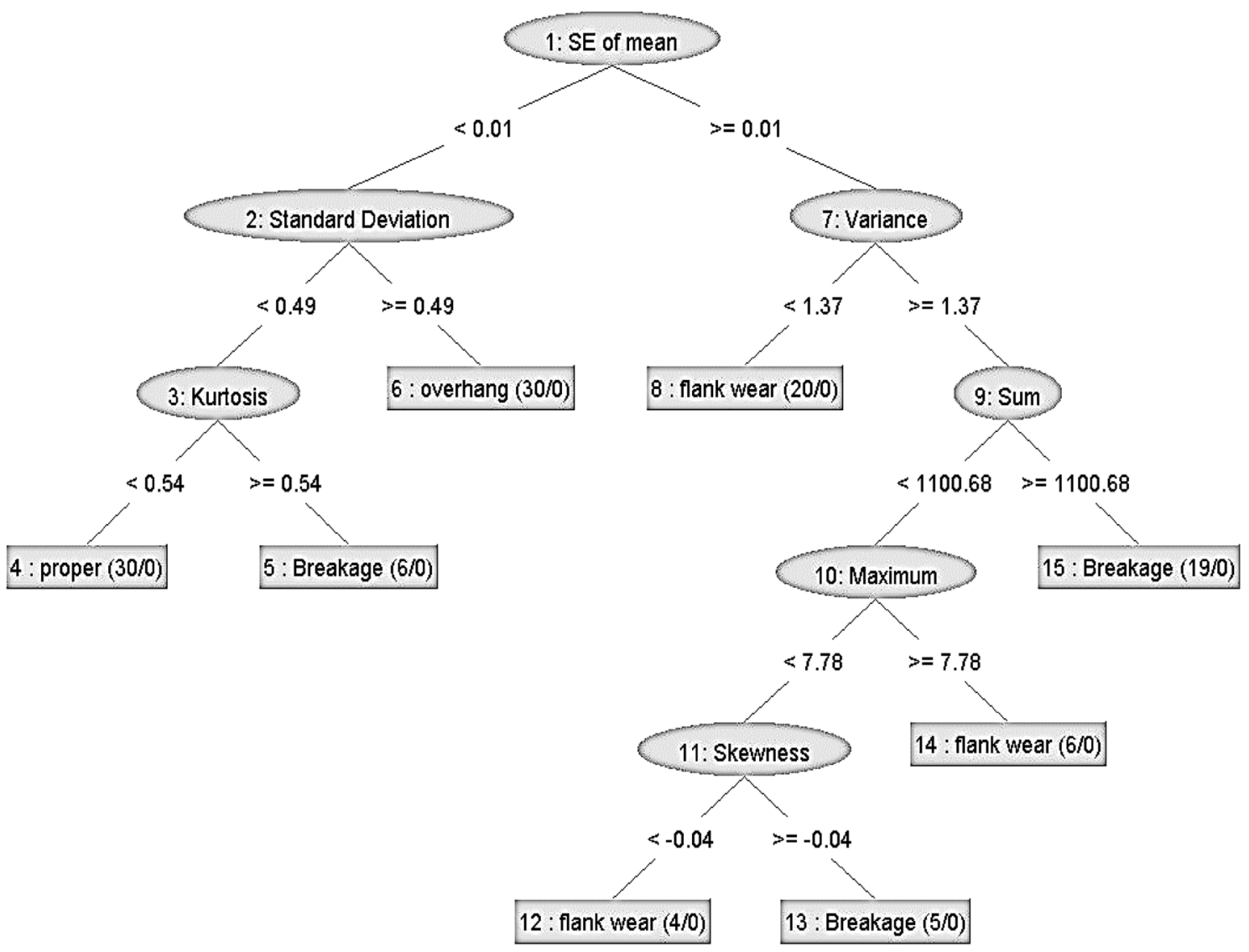

Fig. 6 Decision tree (RT) based on statistical features of vibration data

Table 2 Rotation forest confusion matrix

\begin{tabular}{lrrrl}
\hline$j$ & $k$ & $l$ & $m$ & $\leftarrow$ Classified as \\
\hline 30 & 0 & 0 & 0 & $j=$ proper \\
0 & 30 & 0 & 0 & $k=$ overhang \\
0 & 0 & 26 & 4 & $l=$ Breakage \\
0 & 0 & 4 & 26 & $m=$ flank wear \\
\hline
\end{tabular}

- The third numeric element is the misclassification of 'proper' as 'breakage'.

- The fourth element is the number of 'proper' samples misclassified as an overhang. A similar analysis holds good for the subsequent rows.

In Table 2, the rotation forest algorithm has classified all 30 instances of proper mounted and healthy state of tool correctly. Extended overhang instances are also classified accurately. Breakage: 26 instances are correctly classified as breakage, and the rest four instances are misclassified as flank wear. Flank wear: 26 instances are correctly classified as flank wear, and the rest four instances are misclassified as breakage. This misclassification is due to the similarity in features of breakage and flank wear.

Detailed class-wise accuracy in classifying a tool condition is presented in Table 3. The table presents the fraction of data set of a class being classified correctly and incorrectly. In detailed classification accuracy matrix, a true positive (TP rate) corresponds to 'correct classification accuracy', and for better classification, the value of TP rate should be ' 1 '. Similarly, the false positive rate (FP rate) corresponds to 'accuracy in misclassification'. For better classification accuracy, the value of FP rate should be close to ' 0 '. The TP rate for breakage is less than 1 due to misclassification of breakage into flank wear. Similarly, TP rate for flank wear is also less than 1 due to misclassification of flank wear as breakage.

A comparative study of different classifiers is carried out to choose the best classifier. Table 4 lists the various classifiers' efficiency. Rotation forest algorithm resulted in classification efficiency of $95.0 \%$. J48 and Naïve Bayes were inferior to RT in classifying the tool condition. Hence, rotation forest algorithm is used for classification in the current study. 
Table 3 Detailed class-wise accuracy chart

\begin{tabular}{lllllll}
\hline Class & TP rate & FP rate & Precision & Recall & F-measure & ROC area \\
\hline Proper & 1 & 0 & 1 & 1 & 1 & 1 \\
Overhang & 1 & 0 & 1 & 1 & 1 & 1 \\
Breakage & 0.867 & 0.044 & 0.867 & 0.867 & 0.867 & 0.911 \\
Flank wear & 0.867 & 0.044 & 0.867 & 0.867 & 0.867 & 0.911 \\
\hline
\end{tabular}

Table 4 Classifier's efficiency comparison table

\begin{tabular}{lll}
\hline Sl. no. & Classifier & Efficiency (\%) \\
\hline 1 & Rotation forest & 95.00 \\
2 & Logit boost & 94.17 \\
3 & Random forest & 94.17 \\
4 & Random tree & 93.33 \\
5 & J48 & 92.5 \\
6 & Naïve Bayes & 75.83 \\
\hline
\end{tabular}

\section{Conclusion}

Fault diagnosis of carbide tool insert of rough turning tool was carried out using the vibration signal of the turning process. From the acquired vibration signals, descriptive statistical features were obtained. Significant features which give relevant information about different classes of tool condition were selected by using decision tree algorithm. In view of improving classification accuracy, various classifiers were compared and it was found that rotation forest algorithm classified the instances with $95.00 \%$ accuracy. Hence, a decision tree with rotation forest algorithm can be suggested for condition monitoring or fault diagnosis of a cutting tool.

\section{Compliance with ethical standards}

Conflict of interest The authors declare that they have no conflict of interest.

\section{References}

1. Stephenson DA, Agapiou JS (2016) Metal cutting theory and practice. CRC Press, Boca Raton

2. Siddhpura A, Paurobally R (2013) A review of flank wear prediction methods for tool condition monitoring in a turning process. Int J Adv Manuf Technol 65:371-393. https://doi.org/10.1007/ s00170-012-4177-1

3. Bartarya G, Choudhury SK (2012) State of the art in hard turning. Int J Mach Tools Manuf 53:1-14. https://doi.org/10.1016/j.ijmac htools.2011.08.019

4. Chattopadhyay AK, Chattopadhyay AB, Paul S (2017) Failure of cutting tools and tool life. Manuf Process 2:1-11
5. Munoa J, Beudaert X, Dombovari Z et al (2016) Chatter suppression techniques in metal cutting. CIRP Ann Manuf Technol 65:785-808. https://doi.org/10.1016/j.cirp.2016.06.004

6. Madhusudana CK, Kumar H, Narendranath S (2016) Condition monitoring of face milling tool using K-star algorithm and histogram features of vibration signal. Eng Sci Technol Int J 19:15431551. https://doi.org/10.1016/j.jestch.2016.05.009

7. Madhusudana CK, Budati S, Gangadhar N et al (2016) Fault diagnosis studies of face milling cutter using machine learning approach. J Low Freq Noise Vib Act Control 35:128-138. https ://doi.org/10.1177/0263092316644090

8. Wang J, Ma Y, Zhang L et al (2018) Deep learning for smart manufacturing: methods and applications. J Manuf Syst 48:144-156. https://doi.org/10.1016/j.jmsy.2018.01.003

9. Ma J, Zhang D, Wu B et al (2016) Vibration suppression of thinwalled workpiece machining considering external damping properties based on magnetorheological fluids flexible fixture. Chin J Aeronaut 29:1074-1083. https://doi.org/10.1016/j. cja.2016.04.017

10. Kishore R, Choudhury SK, Orra K (2018) On-line control of machine tool vibration in turning operation using electro-magneto rheological damper. J Manuf Process 31:187-198. https:// doi.org/10.1016/j.jmapro.2017.11.015

11. Ventura CEH, Breidenstein B, Denkena B (2017) Influence of customized cutting edge geometries on the workpiece residual stress in hard turning. Proc Inst Mech Eng Part B J Eng Manuf. https://doi.org/10.1177/0954405416685388

12. Kuljanic E, Sortino M (2005) TWEM, a method based on cutting forces-monitoring tool wear in face milling. Int J Mach Tools Manuf 45:29-34. https://doi.org/10.1016/j.ijmachtool s.2004.06.016

13. Dutta S, Pal SK, Mukhopadhyay S, Sen R (2013) CIRP journal of manufacturing science and technology application of digital image processing in tool condition monitoring: a review. CIRP J Manuf Sci Technol 6:212-232. https://doi.org/10.1016/j.cirpj .2013.02.005

14. Teti R, Jemielniak K, Donnell GO, Dornfeld D (2010) Advanced monitoring of machining operations. CIRP Ann Manuf Technol Adv Monit Mach Oper 59:717-739. https://doi.org/10.1016/j. cirp.2010.05.010

15. Kothuru A, Nooka SP, Liu R (2018) Audio-based tool condition monitoring in milling of the workpiece material with the hardness variation using support vector machines and convolutional neural networks. ASME J Manuf Sci Eng 140:1-9. https://doi. org/10.1115/1.4040874

16. Gangadhar N, Kumar H, Narendranath S, Sugumaran V (2018) Condition monitoring of single point cutting tools based on machine learning approach. Int J Acoust Vib 23:131-137. https ://doi.org/10.20855/ijav.2018.23.21130

17. Zelinski P (2018) The overhang effect. In: Mod. Mach. shop. https ://www.mmsonline.com/articles/the-overhang-effect. Accessed 25 Jun 2018

18. Tiwari $R$ (2000) Rotor systems: analysis and identification. CRC Press, Taylor \& Francis Group, Boca Raton, p 2018

19. Madhusudana CK, Kumar H, Narendranath S (2016) Engineering science and technology, an international journal condition 
monitoring of face milling tool using K-star algorithm and histogram features of vibration signal. Eng Sci Technol Int J 19:15431551. https://doi.org/10.1016/j.jestch.2016.05.009

20. Rodríguez JJ, Kuncheva LI, Alonso CJ (2006) Rotation forest: a new classifier ensemble method. IEEE Trans Pattern Anal Mach Intell 28:1619-1630. https://doi.org/10.1109/tpami.2006.211

21. Cuka B, Kim D-W (2017) Fuzzy logic based tool condition monitoring for end-milling. Robot Comput Integr Manuf 47:22-36. https://doi.org/10.1016/j.rcim.2016.12.009
22. Roiger RJ (2017) Data mining: a tutorial-based primer. CRC Press, Boca Raton

Publisher's Note Springer Nature remains neutral with regard to jurisdictional claims in published maps and institutional affiliations. 